Proc. Indian Acad. Sci. (Earth Planet. Sci.), Vol. 89, Number 2, July 1980, pp. 249-2 59. (C) Printed in India.

\title{
Study of microwave response of coal and sandstone samples
}

\author{
R SINGH*, RAMESH P SINGH ${ }^{\dagger}$ and $\mathrm{K}$ P SINGH \\ Department of Electronics Engineering, Institute of Technology, Banaras Hindu \\ University, Varanasi 221005 , India \\ * Present address: Department of Physics, Post-Graduate College, Ghazipur, India \\ $\dagger$ Department of Geophysics
}

MS received 12 June 1978 ; revised 13 March 1980

\begin{abstract}
Detailed measurements of relative dielectric constant and loss tangent of coal and sandstone samples have been carried out in the X-band of microwave frequency range $(8-10 \mathrm{GHz})$. The effect of moisture, saline and petrol content on the dielectric and loss tangent has been studied. The reflection and transmission coefficient of these samples have been computed. The application of such measurements to geophysical prospecting has been discussed.
\end{abstract}

Keywords. Microwave response; dielectric constant; loss tangent; laboratory measurement.

\section{Introduction}

The response of direct current flow and low frequency electromagnetic waves have been widely used for the exploration of the earth's surface and near surface features (Keller and Licastro 1959; Noritomi 1961; Keller 1963; Watt et al 1963; Parkhomenko 1967; Scott et al 1967; Blomquist 1970; Cook 1970; Lytle 1974) The response of earth-forming constituents to microwaves is fairly sensitive and is capable of revealing the presence of moisture and petroleum as the displacement currents are appreciable at high frequencies, and the dielectric constant of water is an order of magnitude greater than that of most earth-forming constituents. Further, it is established from laboratory measurements that the dielectric constants at microwave frequencies is independent of water salinity (Poley et al 1978). Extensive laboratory measurement of dielectric constant, loss tangent and travel time of microwaves through samples of known composition (Scott et al 1967; Cook 1970; Hipp 1974; Balanis et al 1976) provide valuable information for data interpretation of in situ experiments. Thus, microwave technique proves to be quite useful in exploration of coal mines (Ellerbruch and Adams 1974; Doso 1976; Lytle et al 1976) and dielectric logging is being developed as discriminating tool for isolating water bearing horiz ons from those bearing oil (Freedman and Vogiatzis 1979). 
The aim of this paper is to report values of the dielectric constant and loss tangent of certain earth-forming rocks namely sandstone and coal measured in the laboratory. Microwave responses of dry and wet rock samples as well as of those soaked in petrol and saline water have been carried out. Variation of $\epsilon_{r}$ and $\tan \delta$ in the X-band of microwaves $(8-10 \mathrm{GHz})$ has been studied, which shows that these are quite diagnostic in distinguishing the presence of petrol from that of water, in rock and coal samples. The dielectric constant and loss tangent variation of saline water show comparatively lesser variation with frequency and is found to be independent of the degree of salinity. In addition reflection and transmission coefficients of these samples have also been measured, and their attenuation constants obtained therefrom. Using attenuation constant we have computed the variation of normalised reflected and transmitted powers with the thickness of the sample. The variation of normalised power with thickness reveals the potentiality of microwave probing in determining thickness of coal seams in mines.

\section{Electromagnetic wave propagation through materials}

Electromagnetic wave propagation through homogeneous and isotropic materials is governed by Maxwell's equations

$$
\nabla \times \bar{E}=-\partial \bar{B} / \partial t \text { and } \nabla \times \bar{H}=\sigma \bar{E}+(\partial \bar{D} / \partial t)
$$

where $\sigma$ is the conductivity of the material. $\bar{B}$ and $\bar{D}$ are related to $\bar{H}$ and $\bar{E}$ by the effective permeability and permittivity of the material

$$
\bar{B}=\mu_{\text {ert }} \bar{H} \text { and } \bar{D}=\epsilon_{\text {ort }} \bar{E} .
$$

Considering the time-varying electromagnetic wave of the form $\exp (j \omega t)$, we write

$$
\begin{aligned}
\nabla \times \bar{H} & =j \omega \epsilon_{0}\left(\epsilon^{\prime}-j \frac{\sigma}{\epsilon_{0} \omega}\right) \bar{E}, \\
& =j \omega \epsilon_{0} \epsilon_{r}^{*} \bar{E}
\end{aligned}
$$

where the complex dielectric constant of the medium is

$$
\epsilon_{r}^{*}=\left(\epsilon^{\prime}-j \frac{\sigma}{\epsilon_{0} \omega}\right)=\left(\epsilon^{\prime}-j \epsilon^{\prime \prime}\right)=\epsilon^{\prime}(1-j \tan \delta),
$$

and $\tan \delta=\epsilon^{\prime \prime} / \epsilon^{\prime}$.

$\epsilon^{\prime \prime}$ is the imaginary part of the complex dielectric constant. The wave equation in one-dimensional case can be obtained by eliminating either $\bar{E}$ or $\bar{H}$ from equation (1)

$$
\begin{aligned}
\frac{\partial^{2} \bar{E}_{a}}{\partial z^{2}} & =-j \omega \mu\left(\sigma+j \omega \epsilon^{\prime}\right) \bar{E}_{x}, \\
& =\gamma^{2} \bar{E}_{x},
\end{aligned}
$$


where $\gamma=\left[j \omega \mu\left(\sigma+j \omega \epsilon^{\prime}\right)\right]^{1 / 2}$, is the propagation constant which characterises the nature of the medium. The frequency-dependent propagation constant is written as

$$
\gamma(\omega)=a(\omega)+j \beta(\omega)
$$

$a$ and $\beta$ are attenuation and phase constants respectively. Separating the real and imaginary parts we write

$$
\begin{aligned}
& a(\omega)=\left\{\frac{\omega^{2} \mu \epsilon^{\prime}}{2}\left[\left(1+\left(\sigma / \omega \epsilon^{\prime}\right)^{2}\right)^{1 / 2}-1\right]\right\}^{1 / 2}, \\
& \text { and } \quad \beta(\omega)=\left\{\frac{\omega^{2} \mu \epsilon^{\prime}}{2}\left[\left(1+\left(\sigma / \omega \epsilon^{\prime}\right)^{2}\right)^{1 / 2}+1\right]\right\}^{1 / 2} \text {. }
\end{aligned}
$$

The attenuation constants of rock and coal samples determine the depth of penetration of microwaves. The characteristic distance within the sample through which the incident wave amplitude falls to $70 \%$ is known as skin depth and is expressed as

$$
\delta=1 / \alpha(\omega) .
$$

The travel time of microwaves per meter of the material is written as (Freedman and Vogiatzis 1979

$$
t=\left\{\frac{\epsilon^{\prime}}{2 \epsilon_{0} C^{2}}\left[1+\left(1+\tan ^{2} \delta\right)^{1 / 2}\right]\right\}^{1 / 2},
$$

where $C$ is the velocity of electromagnetic waves in free space in $\mathrm{ms}^{-1}$. The electromagnetic power density for a plane polarised wave propagating through a material is written as

$$
P=E^{2} / \eta,
$$

where $E$ is the electric field, and $\eta_{0}$ is the intrinsic impedance of the medium which is written as

$$
\eta=\left[\frac{\mu}{\epsilon^{\prime}\left[1-j\left(\epsilon^{n} / \epsilon^{\prime}\right)\right]}\right]^{1 / 2} .
$$

The amplitude of the reflection coefficient of the plane parallel reflecting boundary of the bulk sample is

$$
|R|=\left(\eta-\eta_{0}\right) /\left(\eta+\eta_{0}\right)
$$

where $\eta_{0}=\left(\mu_{0} / \epsilon_{0}\right)^{1 / 2}=377$ ohms, is the intrinsic impedance of free space. Similarly the transmission coefficient for the medium is written as

$$
|T|=E_{2} / E_{1}=2 \eta /\left(\eta+\eta_{0}\right) \text {. }
$$

Using the definitions of reflection and transmission coefficients, we define the normalised electromagnetic power reflected from the surface $(1,1)$ of the sample as

$$
P_{\boldsymbol{r}} / P_{i}=\left|\left(\eta-\eta_{0}\right) /\left(\eta+\eta_{0}\right)\right|^{2} .
$$


Likewise, the normalised reflected power from the face $(2,2)$ of the sample, accounting for the finite attenuation of the electromagnetic power is written as

$$
P_{t} / P_{i}=\left|\frac{2 \eta_{0}}{\eta+\eta_{0}}\right|\left|\frac{2 \eta}{\eta+\eta_{0}}\right| \exp (-4 a x)
$$

where $x$ is the propagation distance within the sample.

\section{Sample preparation}

Coal and sandstone samples for present measurements were cut to size and their surfaces were made fine by grinding. The cross-section of these samples for the $\mathrm{X}$-band measurements were $1.00 \times 2.20 \mathrm{~cm}$ which was the same as the dimension of the rectangular waveguide. The samples thus prepared were placed in a dry nitrogen atmosphere for more than $48 \mathrm{hr}$ and were finally stored at room atmosphere for more than $24 \mathrm{hr}$ before measurement. For preparing non-dry samples, they were soaked overnight in distilled water, saline of strength $0.7 \mathrm{~N}$, and petrol as desired. They were weighed with the help of a chemical balance after being thoroughly dried. The difference in weight between soaked and dry samples gives the moisture content, petrol content or salinity defined as

$\left.\begin{array}{l}\text { Moisture } \\ \begin{array}{l}\text { Petrol } \\ \text { Saline }\end{array}\end{array}\right\}$ content $=\frac{\text { weight of soaked sample }- \text { weight of dry sample }}{\text { weight of dry sample }} \times 100 \%$

Repeated measurements of the dielectric constant with varying frequency in the $\mathrm{X}$-band were carried out on each sample.

\section{Experimental method}

Various methods have been used for the determination of dielectric properties of material at microwave frequencies (Sucher and Fox 1963; Balanis et al 1976) the important ones being the transmission line method, the cavity perturbation method and the resonance method. In these methods the transmission lines or cavities are either partially or completely filled with an unknown dielectric material. These methods are therefore, directly suited for in situ measurements.

In the present study we measured the dielectric constant and loss tangent of especially cut and prepared samples in the X-band using the slotted waveguide transmission line technique. In the rectangular waveguide, the $\mathrm{TE}_{01}$ modes were dominantly excited and corresponding measurements were made. The wellknown two-point method of measurement (Sucher and Fox 1963) involving transcendental equation (21) was followed throughout this study which is capable of precisely measuring both the dielectric constant and the loss tangent.

The basic arrangement of the experimental set-up is shown in figure 1 (a). The slotted line is adjacent to a short-circuited length of the waveguide. In the first part of the measurement the sample is inserted into the waveguide so that one end of the sample touches the short-circuited end of the waveguide. The 


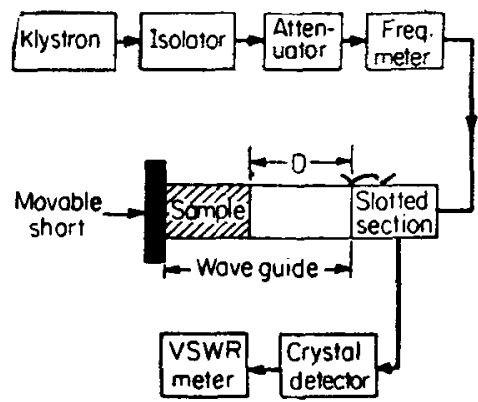

(a)

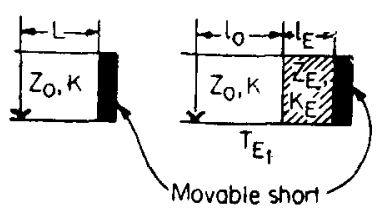

(b)

Figure 1. Basic arrangament of the experimental set-up.

second part of the measurement is carried out by removing the sample and keeping the short-circuited length of the waveguide empty. Let $D_{R}$ be the position of the probe showing voltage minimum when the short-circuited waveguide is empty. This distance from the short-circuited end is denoted by $l_{R}+l_{\epsilon}$. Similar positions of the voltage minimum are located with one end of the sample touching the short-circuited end of the waveguide. This position of the voltage minimum probe is denoted by $D$ and its distance from the short-circuited end of the waveguide is written as $l+l_{\varepsilon}$ which is shown in figure $1(\mathrm{~b})$. The matched impedance, when the hollow waveguide is terminated by a movable metallic short as shown in figure 1 (b) can be written as

$$
Z_{0} \tan k\left(l_{R}+l_{e}\right)=0 \text {. }
$$

In the case of the waveguide being partially filled with the dielectrics ample, the matched impedance at the interface of the air and dielectric is written as

$$
Z_{0} \tan k l=-Z_{\epsilon} \tan k_{e} l_{e} .
$$

For two consecutive voltage minima of the probe, the impedance equation is written as

$$
\tan k\left(D_{R}-D+l_{\epsilon}\right)=\tan k\left[\left(l_{R}+l_{\varepsilon}\right)-l\right] .
$$

Expanding the right side of equation (20) and substituting from equation (18)-(19) we obtain,

$$
\frac{\tan k\left(D_{R}-D+l_{\epsilon}\right)}{k l_{\epsilon}}=\frac{\tan k_{\epsilon} l_{\epsilon}}{k_{\epsilon} l_{\epsilon}}=\frac{\tan \chi}{\chi} .
$$

This is a transcendental equation which yields infinite values of $\chi$. In order to ascertain the dielectric constant of an unknown sample, the parameters $D_{R}, D, l_{e}$ and $k_{e}$ are measured for two pieces of different lengths at a fixed frequency; and from these values, $\chi$ are obtained using equation (21). The variation of $\epsilon^{\prime}$ with $\chi$ values are computed from the following relation

$$
\epsilon^{\prime}=\left(\chi \lambda_{0} / 2 \pi l\right)^{2}+\left(\lambda_{0} / \lambda_{c}\right)^{2}
$$


The variation of the dielectric constant with $\chi$, thus obtained for two lengths of a rock sample are then plotted and the intersection of the two curves gives the correct value of the dielectric constant of the material under investigation (Sucher and Fox 1963).

Further, the loss tangent of the dielectric material has been computed with the help of the equation

$$
\tan \delta=\left(\triangle X_{s}-\triangle X_{a}\right) / \epsilon^{\prime}\left(\lambda_{0} / \lambda_{g}\right)^{2}
$$

where $\lambda_{0}=\lambda_{0} /\left[1 /\left(\lambda_{0}-\lambda_{o}\right)^{2}\right]^{1 / 2}, \Delta X_{s}$ and $\Delta X_{a}$ denote the distances for a fixed voltage standing wave ratio (VSWR) on either side of the minimum. The values of $\triangle X_{a}$ and $\triangle X_{a}$ have been measured with the help of a slotted line by using the well-known double minimum method where $\lambda_{c}=2 a$, $a$ being the width of the rectangular waveguide.

\section{Results and discussions}

The relative dielectric constant of coal and sandstone samples have been measured using the method described in $\$ 4$. Variation of $\epsilon^{\prime}$ with frequency for sandstone is shown in figure 2. It is found to be almost constant in the entire X-band frequency range. Decreasing trend in dielectric constant at higher frequency is seen for dry, wet and petrol-soaked samples of sandstone. The dielectric constant of saline-soaked sandstone sample is seen to be comparatively much higher. The dielectric constant of saline-soaked sample depicts an increasing trend toward the higher frequency end. The dry sample forms the basic trend of dielectric constant variation and the filling of the pore spaces by water, petrol and saline water control the resulting nature of variation with frequency. Similar frequency variations for coal samples are shown in figure 3 . The trend of

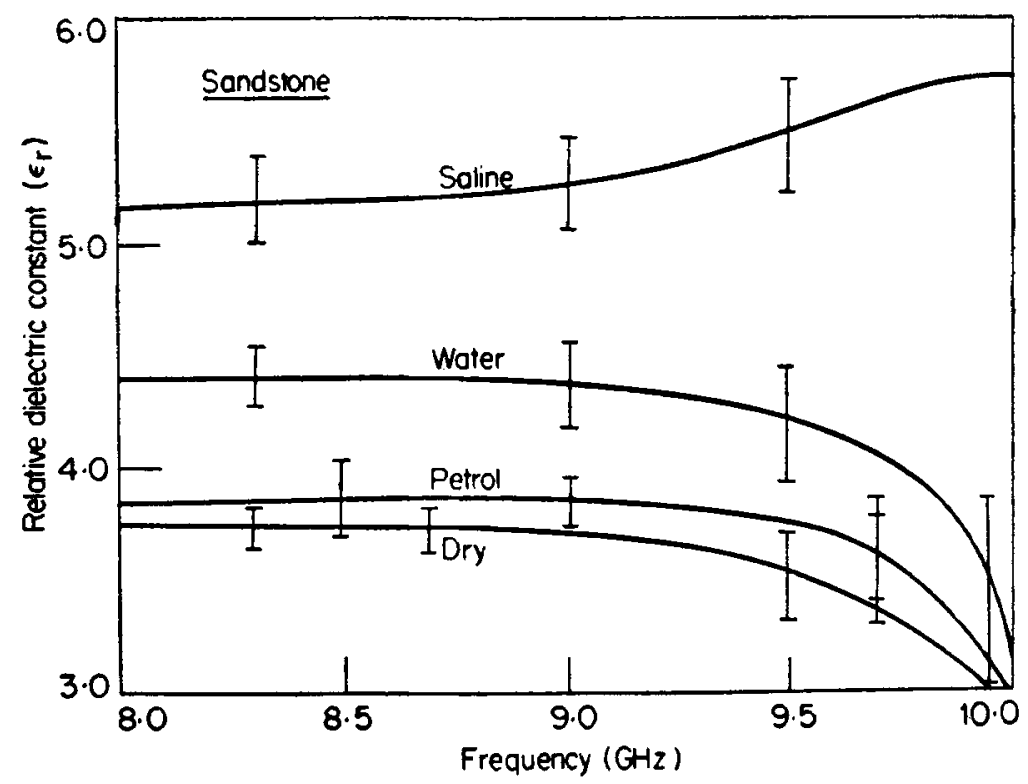

Figure 2. Variation of relative dielectric constant of sandstone with frequency. 
frequency variation depicted by the dry coal sample is seen in the wet and saline soaked coal samples. The petrol-soaked sample of coal depicts an anomalous behaviour and is seen to exhibit smaller dielectric constant as compared with the dry sample. The variation of $\tan \delta$ with frequency is shown for the same set of coal samples (figures 4 and 5). The $\tan \delta$ variation depicts the general trend of increase with increasing frequency. The measurements of $\tan \delta$ are comparatively less accurate as compared to those of dielectric constant. The peak of $\tan \delta$ corresponds to the maximum variation of dielectric constant with frequency. For sandstone, the peak of $\tan \delta$ seems to be much beyond $10 \mathrm{GHz}$ (figure 4), whereas, for coal the peak formation is seen to lie very close to $10 \mathrm{GHz}$ (figure 5). The one-to-one correspondence between dielectric constant and $\tan \delta$ variation is not very satisfactory which depicts that the dielectric constant and loss tangent

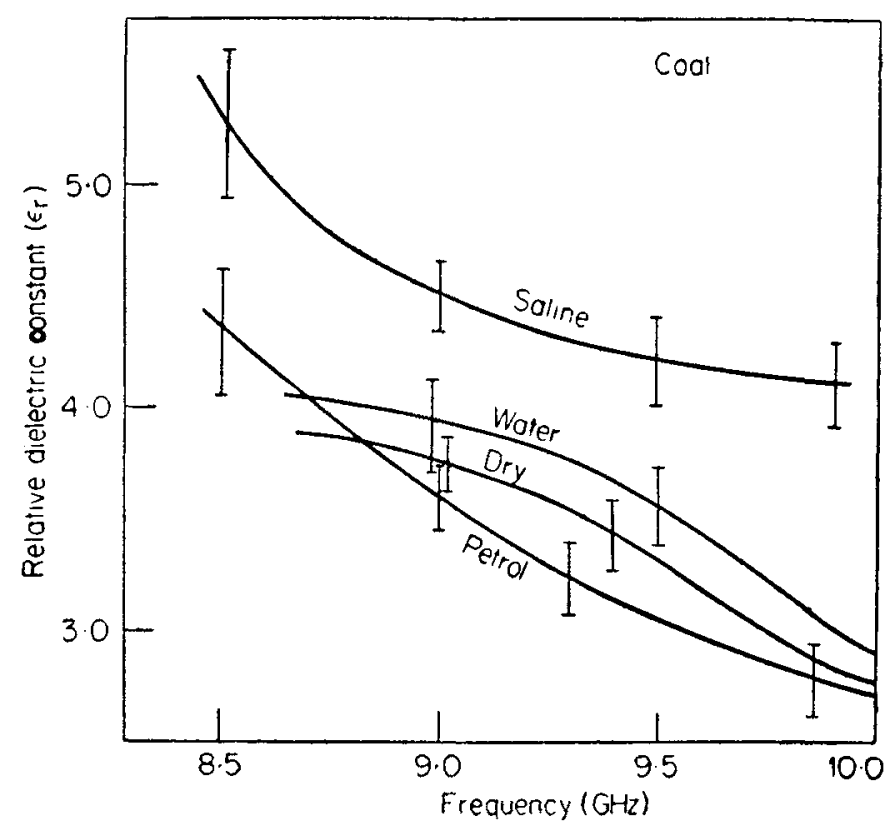

Figure 3. Variation of relative dielectric constant of coal sample with frequency.

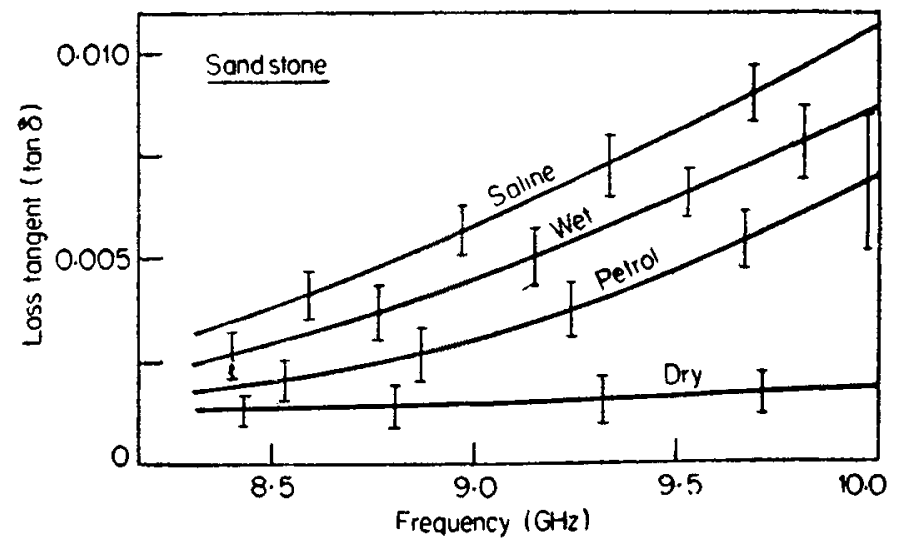

Figure 4. Variation of loss tangent of sandstone sample with frequency.

P. (A) -14 
cannot be measured accurately using the same method. The loss tangent arises due to non-linear effects and is difficult to measure with desired accuracy.

The variation of attenuation constant of microwave signals with frequency has been computed by accounting for the frequency variation of dielectric constant and conductivity of the same set of sandstone and coal samples. The computed results are shown in figure 6 . In general, the attenuation constant is seen to

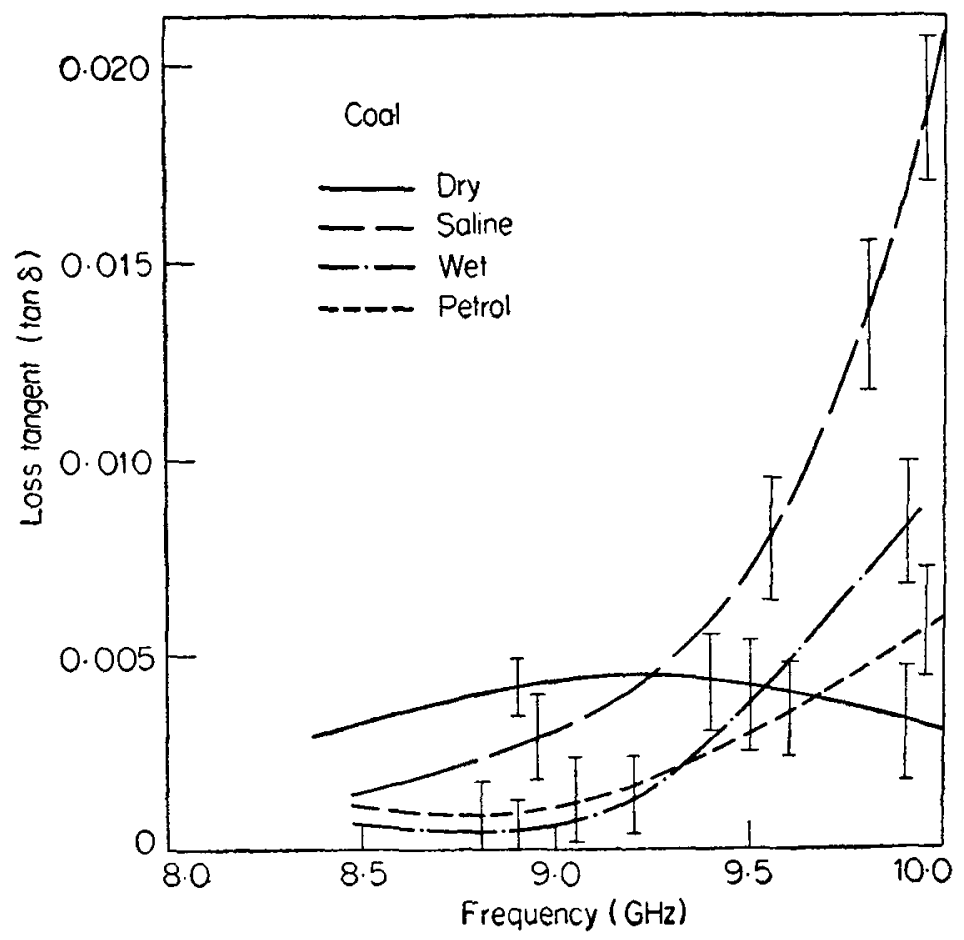

Figure 5. Variation of loss tangent of coal sample with frequency.

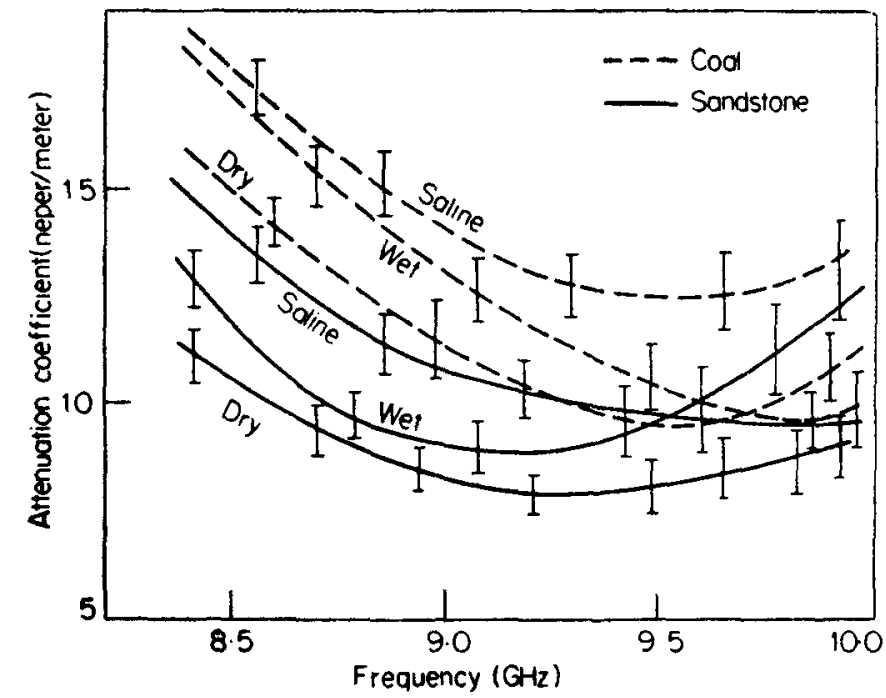

Figure 6. Variation of attenuation constant with frequency in the coal and sand stone sample. 
decrease with increasing frequency. The variation of a for dry samples of coal and sandstone is shown along with similar results for water and saline-soaked samples. It is shown that the water and saline-soaked samples result into increased attenuation constant in the entire $\mathrm{X}$-band. The difference from one curve to another curve depends on the moisture and saline content of the sample but in each case the attenuation constant is seen to increase. Using the maximum and minimum values of calculated $a$, we find that the depth of penetration of microwaves through rocks and coal varies from 5 to 12 .

The reflection and transmission coefficients for chosen samples of sandstone and coal have been measured and their variations with frequency are shown in figures 7 and 8 . The reflection coefficients for the samples show a small decrease with increasing frequency, whereas transmission coefficients for these samples are almost constant with frequency variation. For each of these samples the sum of $|R|$ and $|T|$ is fairly small as compared to unity. This clearly shows that a significant amount of electromagnetic energy is scattered at the reflecting and transmitting interfaces and dissipated in the sample. For example the sandstone sample soaked in saline water, shows the largest reflection coefficient and the largest transmission coefficient which implies that the electromagnetic energy absorbed is minimum. This is probably due to the fact that the saline soaked sample becomes highly conducting as compared to other samples. The measured $|R|$ and $|T|$ for coal samples with moisture, petrol and saline water contents are shown in figure 8 . Taking into account the frequency variation of attenuation constant, the variation of normalised reflected power from the back face of the samples for various thicknesses of the sample has been computed which is shown in figure 9. The transmitted power is seen to decrease steadily with increasing thickness of the sample. The change in the signal levels of microwave

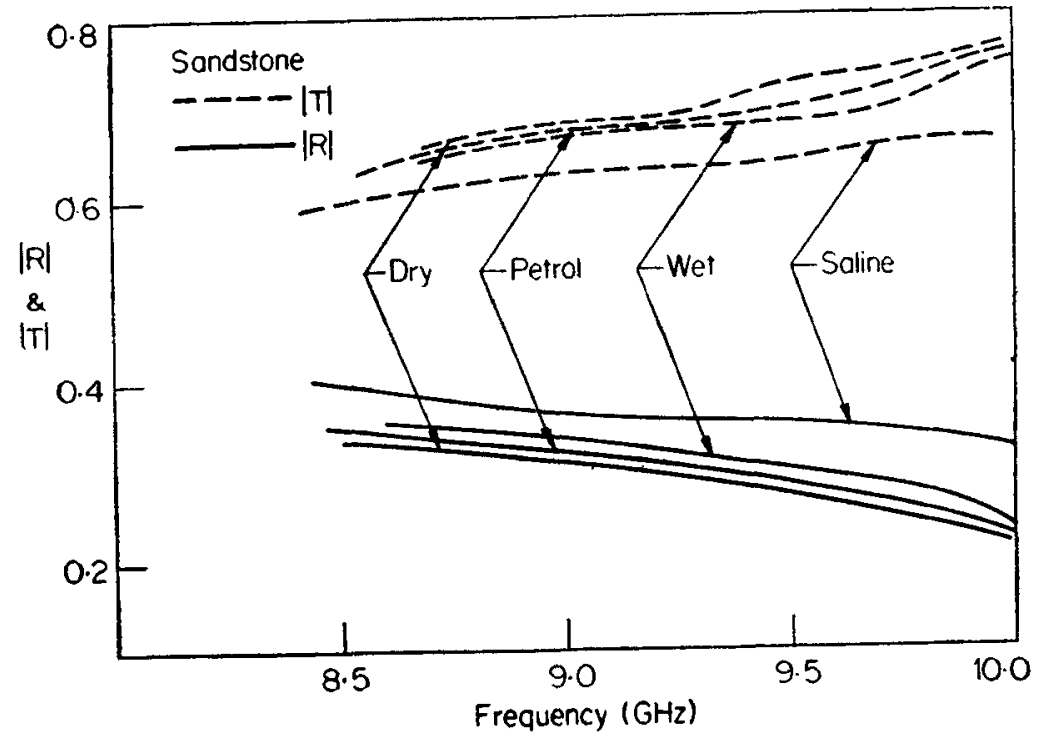

Figure 7. Variation of reflection and transmission coefficient of sandstore sample with frequency. 


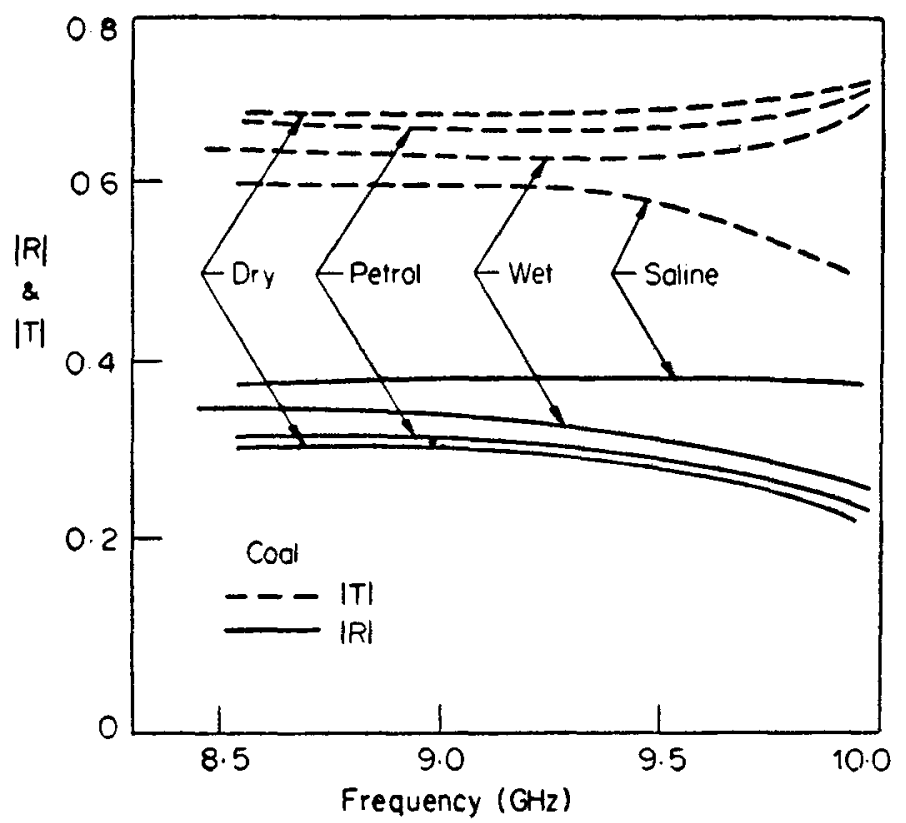

Figure 8. Variation of reflection and transmission coefficient of coal sample with frequeney.

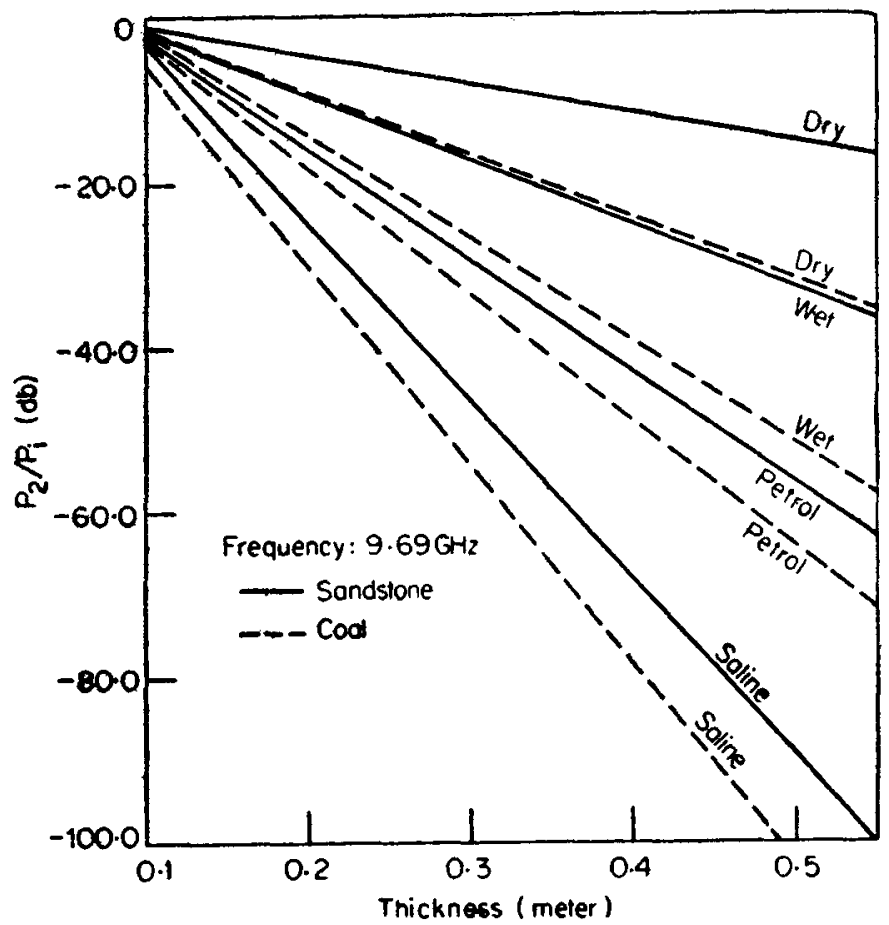

Figure 9. Variation of relative power density with depth. 
power reflected from two interfaces of coal sample is capable of revealing certain features of the coal sample.

\section{Conclusion}

The master curves prepared from extensive measurement of dielectric constant and loss tangent with varying moisture, petrol and salinity content can be used as a quick aid for inversion and diagnostic studies on new samples. The measurement of reflection and transmission coefficients reveal the nature of rock and coal samples. Laboratory measurements of various physical parameters may provide important clue in the analysis of geophysical exploration data.

\section{Acknowledgements}

We are grateful to Professor R N Singh for his keen interest in this work. Two of us (RS and RPS) are thankful to University Grants Commission, New Delhi, for financial support. We are thankful to Professor Jagdeo Singh and Dr T Lal for helpful discussions. Thanks are also due to Dr Raghunath for helping us in computation.

\section{References}

Balanis C A, Rice W S and Smith N S 1976 Radio Sci. 11413

Blomquist A 1970 Proc. Int. Meeting on Radioglaciol. p. 54

Cook J C 1970 Geophysics 351079

Davis J L and Annan A P 1977 Can. J. Remote Sensing 376

Doso H W 1976 Can. J. Earth Sci. 3917

Ellerbruch D A and Adams J W 1974 Tech. Rep. NBSIR 74387

U.S. National Bureau of Standards, Boulder, Colorado

Freedman R and Vogiatzis J P 1979 Geophysics 44969

Hipp J E 1974 Proc. IEEE 6298

Keller G V 1963 IEEE Trans. Antennas. Propag. AP 11344

Keller G V and Licastro P H 1959 U.S. Geol. Surv. Bull. 1052 H 257

Lytle R J 1974 IEEE Trans. Geosci. Elect. GE 1281

Lytle R J, Laine E F, Lager D L and Okada J T 1976 Radio Sci. 11285

Noritomi K 1961 J. Min. Coll. Akita Univ. 1A 27

Parkhomenko E O 1967 Electrical properties of rocks (New York: Pergamon press)

Poly J Ph, Nooteboom J J and dewaal P J 1978 Log. Analyst. 198

Scott J H, Carroll R D and Cunningham D R 1967 J. Geophys. Res. 725101

Sucher M and Fox J 1963 Hand-book of microwave measurement (New York : Polytechnic) 2 Chapter 9

Watt A D et al 1963 Proc. IEEE 51897 